Supplement of Atmos. Chem. Phys., 21, 7409-7427, 2021

https://doi.org/10.5194/acp-21-7409-2021-supplement

(C) Author(s) 2021. CC BY 4.0 License.

(c) (1)

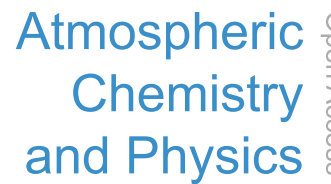

Supplement of

\title{
Secondary aerosol formation alters CCN activity in the North China Plain
}

Jiangchuan Tao et al.

Correspondence to: Jiangchuan Tao (taojch@jnu.edu.cn) and Nan Ma (nan.ma@jnu.edu.cn)

The copyright of individual parts of the supplement might differ from the article licence. 


\section{Inversion method of Size-resolved Particle Activation Ratio (SPAR):}

When the DMA is charged with a negative voltage, those aerosols with a small range of electrical mobility $\left(Z_{P}\right)$ can pass through the DMA. When the scan diameter is set as Dpi for the singly charged particles and the respective voltage of DMA is $\mathrm{V}_{\mathrm{i}}(\mathrm{i}=1,2, \ldots, \mathrm{I})$, aerosol particles with an electrical mobility of $Z_{p, i}(i=1,2, \ldots, I)$ can pass through the DMA and the observed $\mathrm{N}_{\mathrm{CCN}}$ by CCN counter can

6 be expressed as:

$$
R_{i}=\int_{0}^{\infty} G(i, x) A(x) n(x) d x
$$

where $\mathrm{x}$ is the scale parameter with the definition of $\mathrm{x}=\log \left(\mathrm{D}_{\mathrm{pi}}\right)$; $\mathrm{A}(\mathrm{x})$ is the SPAR of a single particle for scale parameter $\mathrm{x}$; and $\mathrm{n}(\mathrm{x})=\mathrm{dN} / \mathrm{d} \log \mathrm{D}_{\mathrm{p}}$ is aerosol PNSD that is the multiple charging corrected results from the measured aerosol PNSD. We define the kernel function $\mathrm{G}(\mathrm{i}, \mathrm{x})$, which is crucial to the

11 algorithm, as:

$$
G(i, x)=\sum_{v=1}^{\infty} \phi(x, v) \Omega(x, v, i)
$$

13 where $\phi(x, v)$ is the probability of particles that are charged with $\mathrm{v}$ charges at the scale parameter of $14 \mathrm{x}$ (Wiedensohler, 1988). Transfer function $\Omega(x, v, i)$ is the probability of particles that can pass 15 through the DMA with $\mathrm{v}$ charges at the scale parameter $\mathrm{x}$ (Knutson and Whitby, 1975). In this study, 16 the maximum value of $v$ is 10 .

The multiple charging corrections can be expressed as computing the $\mathrm{A}\left(\mathrm{x}_{\mathrm{i}}^{*}\right)$, in which $\mathrm{x}_{\mathrm{i}}^{*}$ is the predetermined scale parameter from the DMA. To get the numerical integration results of Eq. (9), the range of the diameter is $\left[x_{i n t, 1}, x_{i n t, J}\right]$ and the diameter interval that is $1 / 50$ of the measured diameter is used. For $x_{i n t, 1}$, its mobility is the $50 \%$ higher than the mobility of $x_{1}{ }^{*}$ with single charge. For $x_{i n t, J}$, its mobility is the $50 \%$ higher than the mobility of $x_{I}{ }^{*}$ with ten charges. Thus, Eq. (S2) can be written as:

$$
R_{i}=\int_{x_{\mathrm{int}, 1}}^{x_{\mathrm{int}, J}} G(i, x) A(x) n(x) d x=\Delta x_{\mathrm{int}} \sum_{j=1}^{J} \beta_{j} G\left(i, x_{\mathrm{int}, j}\right) A\left(x_{\mathrm{int}, j}\right) n\left(x_{\mathrm{int}, j}\right)
$$

23 where $\beta_{j}=\left\{\begin{array}{l}0.5, j=1, J \\ 1, \text { otherwise }\end{array}, \mathrm{x}_{\mathrm{int}, \mathrm{j}}\right.$ is the $\mathrm{jth}(j=1,2, \ldots, J)$ parameter that locates at the parameter $\mathrm{x}_{\mathrm{i}}$ and $24 \mathrm{x}_{\mathrm{i}+1}$, and $A\left(x_{\mathrm{int}, j}\right), j=1,2, \ldots, J$ is SPAR at scale parameter $\mathrm{x}_{\mathrm{int}, \mathrm{j}}$, which is expressed as the linear 25 interpolation of the values at the measured diameters:

$$
A\left(x_{\mathrm{int}, j}\right)=A\left(x_{i(j)}^{*}\right)+P_{i(j)}\left(x_{\mathrm{int}, j}-x_{i(j)}^{*}\right)
$$

27 where $\mathrm{Pi}$ is the slope of the linear interpolation result of the five diameters that are nearest to the 
Then by considering

$$
H_{i j}=\beta_{j} \Delta x_{\mathrm{int}} G\left(i, x_{\mathrm{int}, j}\right) n\left(x_{\mathrm{int}, j}\right)
$$

31 the equation (S3) can be rewritten as:

$$
R_{i}=\sum_{j=1}^{J} H_{i j} A\left(x_{\mathrm{int}, j}\right)
$$

33 then

$$
R_{i}=\sum_{j=1}^{J} H_{i j}\left[A\left(x_{i(j)}^{*}\right)+P_{i(j)}\left(x_{\mathrm{int}, j}-x_{i(j)}^{*}\right)\right]
$$$$
=\sum_{j=1}^{J} H_{i j} A\left(x_{i(j)}^{*}\right)+\sum_{j=1}^{J} H_{i j} P_{i(j)} x_{\mathrm{int}, j}-\sum_{j=1}^{J} H_{i j} P_{i(j)} x_{i(j)}^{*}
$$$$
=\sum_{k=1}^{I}\left(\sum_{j=1}^{J} H_{i j} \delta(i(j)-k)\right) A\left(x_{k}^{*}\right)
$$$$
+\sum_{k=1}^{I}\left(\sum_{j=1}^{J} H_{i j} x_{\mathrm{int}, j} \delta(i(j)-k)\right) P_{k}
$$$$
-\sum_{k=1}^{I}\left(\sum_{j=1}^{J} H_{i j} \delta(i(j)-k)\right) P_{k} x_{k}^{*}
$$$$
=\sum_{k=1}^{I} Q_{i k} A\left(x_{k}^{*}\right)+\sum_{k=1}^{I} T_{i k} P_{k}-\sum_{k=1}^{I} Q_{i k} P_{k} x_{k}^{*}
$$

40 where the Dirac Function is:

$$
\delta(x)=\left\{\begin{array}{l}
0, x \neq 0 \\
1, x=0
\end{array}\right.
$$

42 thus

$$
Q_{i k}=\sum_{j=1}^{J} H_{i j} \delta(i(j)-k)
$$

$$
T_{i k}=\sum_{j=1}^{J} H_{i j} x_{\mathrm{int}, j} \delta(i(j)-k)
$$

45 by letting the

$$
S_{i}=R_{i}-\sum_{k=1}^{I} T_{i k} P_{k}+\sum_{k=1}^{I} Q_{i k} P_{k} x_{k}^{*}
$$

47 this equation is then expressed as

$$
S_{i}=\sum_{k=1}^{I} Q_{i k} A\left(x_{k}^{*}\right)
$$

49 or

51 where $\mathrm{S}$ and $\mathrm{A}$ are $\mathrm{I} \times 1$ vectors and $\mathrm{Q}$ is an $\mathrm{I} \times \mathrm{I}$ matrix. This matrix can be solved by using the non-

52 negative least square method. Finally, the A(x) can be determined and the corresponding size-resolved 53 SPAR that is multiple charging corrected can be calculated. 


\section{Reference:}

56 Wiedensohler, A.: An approximation of the bipolar charge distribu- tion for particles in the submicron 57 size range, J. Aerosol Sci., 19, 387-389, 1988.

58 Knutson, E. O. and Whitby, K. T.: Aerosol classification by electric mobility: apparatus, theory, and 59 applications, Jo. Aerosol Sci., 6, 443-451, 1975.

60 


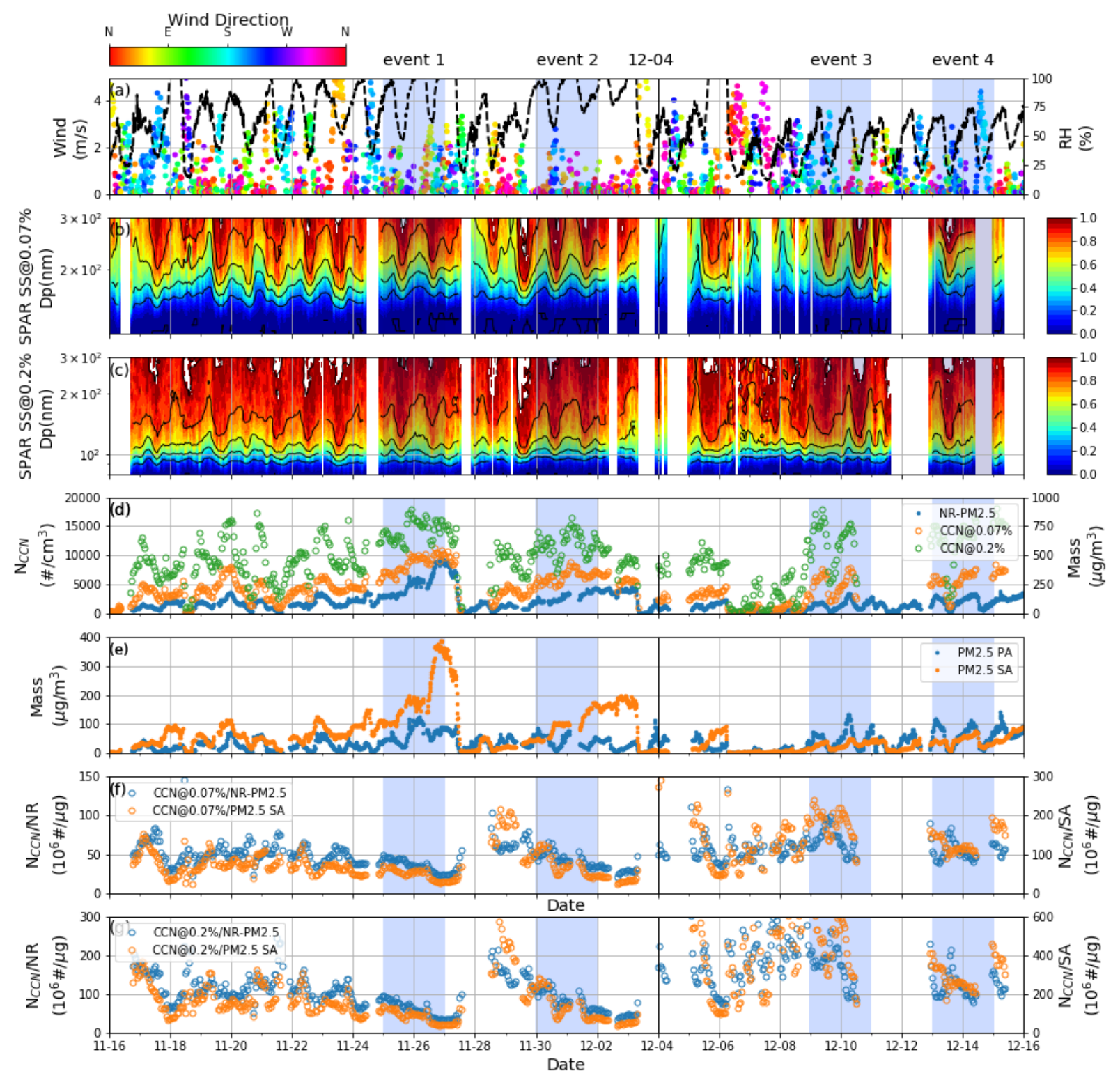

62 Fig S1. Overview of the measurements during the campaign: (a) dots represent wind speed with color

63 indicating wind direction, and black lines represent RH; (b) SPAR under SS of 0.07\%; (c) SPAR under

64 SS of $0.2 \%$; (d) blue, green and yellow dots represent NCCN under SS of $0.07 \%$ and $0.2 \%$, and mass

65 concentration of NR-PM2.5, respectively; (e) blue and yellow dots represent mass concentration of

66 PM2.5 PA and PM2.5 SA respectively; (f) blue and yellow dots represent ratio between NCCN at SS

67 of $0.07 \%$ and mass concentration of NR-PM2.5 and PM2.5 SA, respectively. (g) blue and yellow dots

68 represent ratio between NCCN at SS of 0.2\%and mass concentration of NR-PM2.5 and PM2.5 SA,

69 respectively. There were four events with significant enhancements of NCCN during the blue shaded

70 periods. 


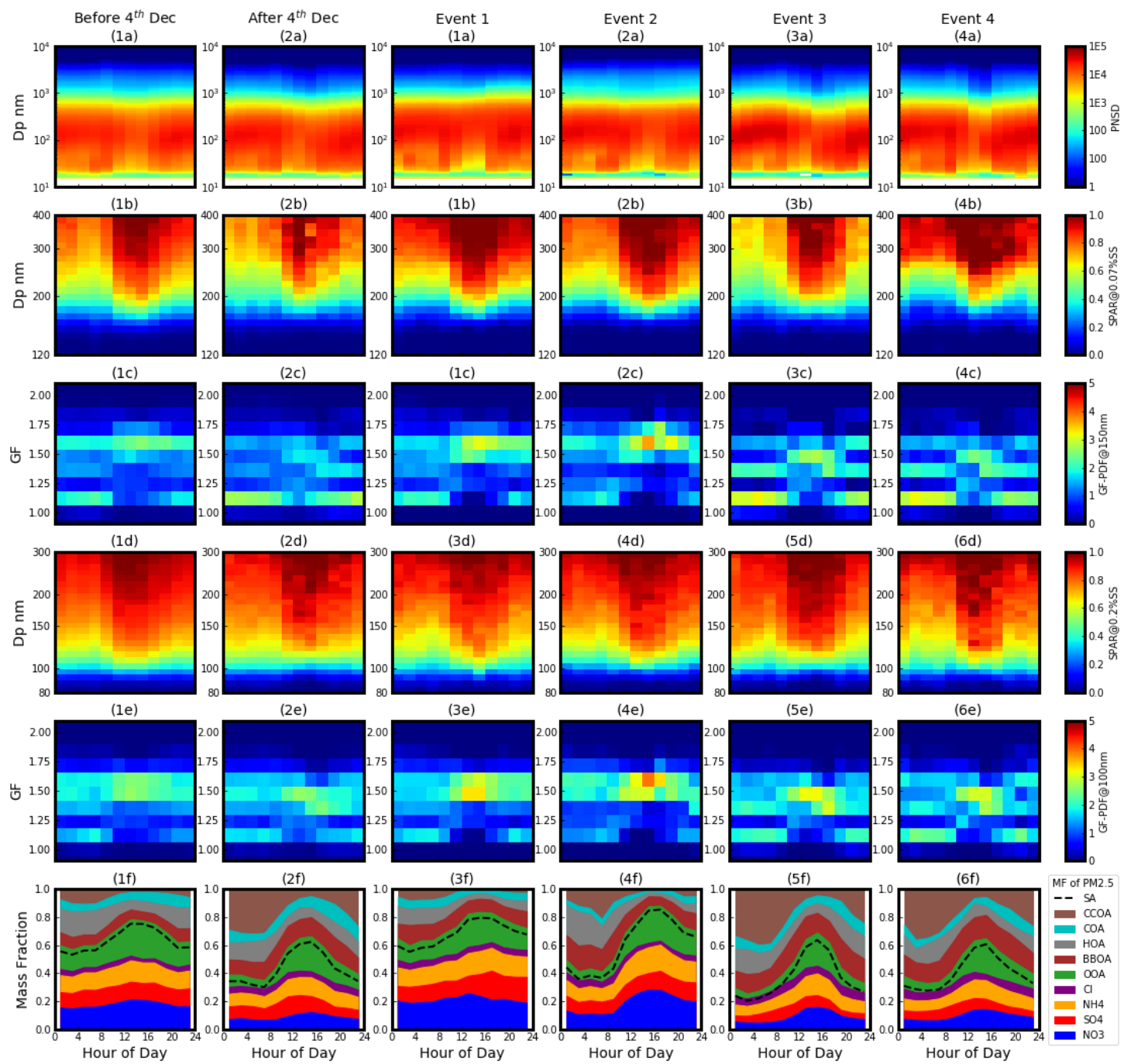

72 Fig S2. Diurnal variation of (a) PNSD, (b) SPAR at SS of $0.07 \%$, (c) GF-PDF at 150nm, (d) SPAR at

73 SS of $0.2 \%$, (e) GF-PDF at 100nm and (f) mass fraction of different PM2.5 chemical species during

74 high RH periods before $4^{\text {th }} \operatorname{Dec}(1)$ low RH periods after $4^{\text {th }} \operatorname{Dec}(2)$ and the four events (3-6), including

75 OA factors: hydrocarbon-like OA (HOA), cooking OA (COA), biomass burning OA (BBOA), coal

76 combustion OA (CCOA), and oxygenated OA (OOA). 

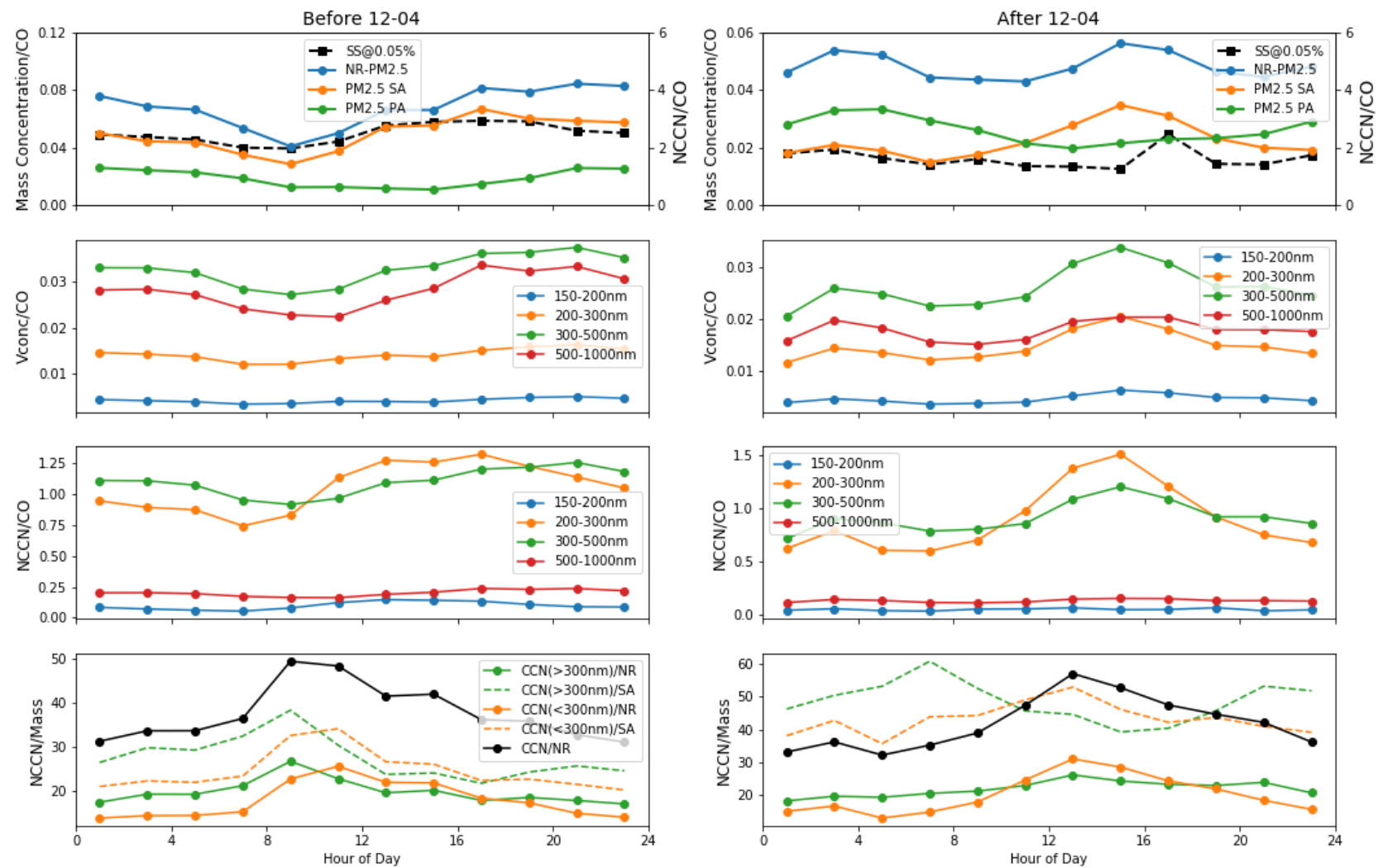

78 Fig S3. The same as Figure 4 but for periods before and after 12-04.

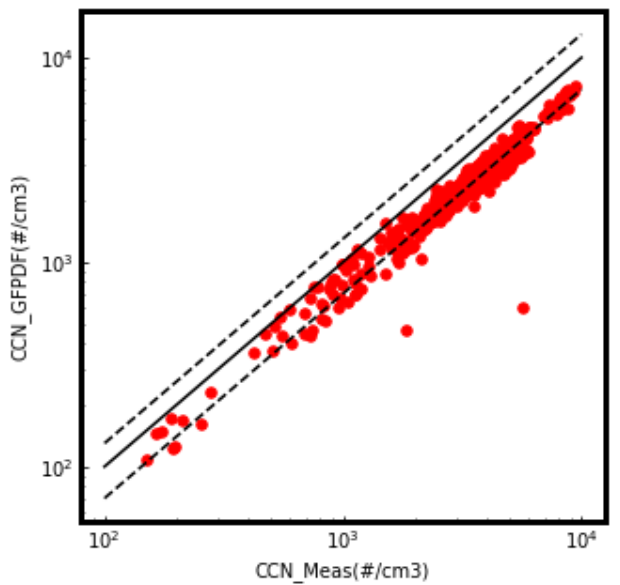

80 Figure S4. Comparison between the calculated NCCN based on GF-PDF and the measured NCCN. 

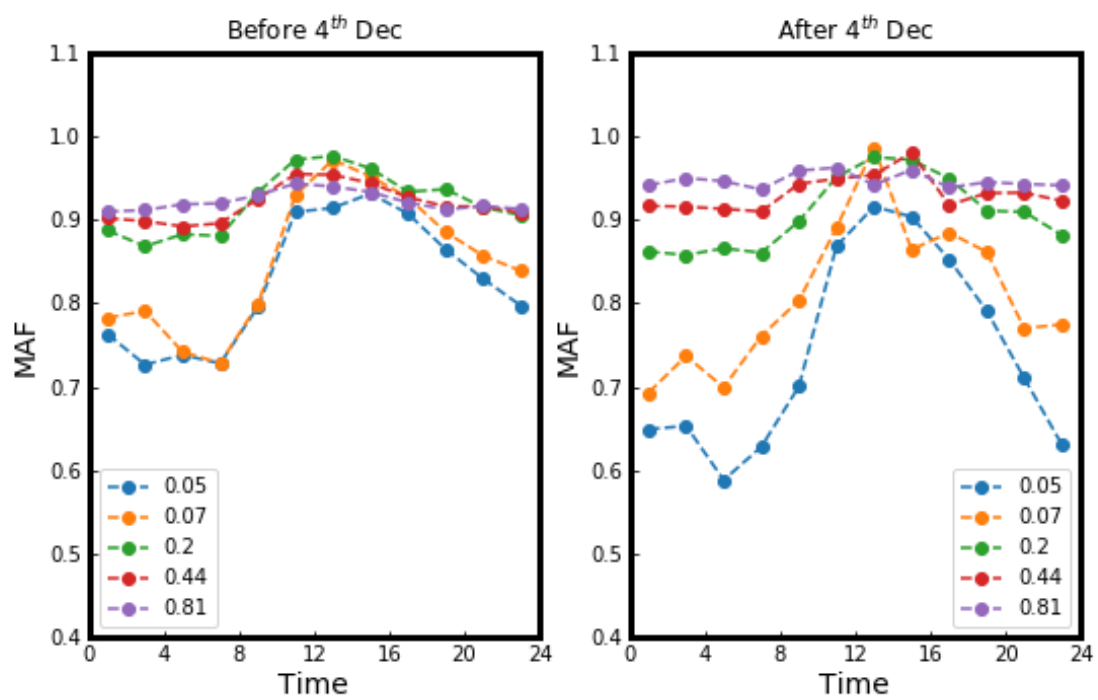

83 Figure S5. Diurnal variations of MAF at the five measured SSs (indicated by different colors) during 84 the high (left) and low (right) RH periods. 